\title{
See-saw mechanism and four light neutrino states
}

\author{
M.Czakon, J.Gluza and M.Zrałek \\ Department of Field Theory and Particle Physics \\ Institute of Physics, University of Silesia, Uniwersytecka 4 \\ PL-40-007 Katowice, Poland
}

\begin{abstract}
A formal proof is given that in a see-saw type neutrino mass matrix with only two neutrino mass scales $\left(m_{D} \ll m_{R}\right)$ and the maximal rank of $m_{R(D)}$ we can not get a fourth light sterile neutrino.
\end{abstract}

It has been known for long that extended gauge group models, such as $S O(10)$ 回 or $S U(2)_{L} \times S U(2)_{R} \times U(1)_{B-L}$ [2], naturally develop a see-saw type neutrino mass matrix. Namely $\left(m_{D}\right.$ is a $3 \times n_{R}$ matrix and $m_{R}$ is a $n_{R} \times n_{R}$ matrix)

$$
m_{\nu}=\left(\begin{array}{cc}
0 & m_{D} \\
m_{D}^{T} & m_{R}
\end{array}\right)
$$

coupled with a large scale difference between $m_{D}$ and $m_{R}$, yields a mass spectrum containing three light Majorana neutrinos, effectively described by

$$
m_{\text {light }} \simeq m_{D}^{T} m_{R}^{-1} m_{D} .
$$

This leads to two different light $\Delta m^{2}$ mass scales, enabling one to understand the solar and the atmospheric neutrino anomalies 
According to the current data (now at $99 \%$ c.l. [4]) a fourth sterile neutrino is not necessary to explain the Superkamiokande data. Suppose however that the LSND experiment [3] is confirmed and we wish also to explain its data in a language of neutrino oscillation phenomena. We then have to introduce a fourth light neutrino of sterile nature (due to the invisible width measurement at LEP). To avoid fine tunings of parameters and still have another light neutrino, one requires the theory to have additional properties, like approximate horizontal symmetry [5], exact parity symmetry [6], a discrete $Z_{5}$ symmetry [7], global $S_{3} \times Z_{2}$ symmetry [8], or even additional gauge $\left(S U(2)_{S}\right)$ symmetry [9]. For more examples see [10]. Some phenomenological considerations have also appeared (see e.g. [11). Interestingly enough, a see-saw type mass matrix Eq. (1) can also lead to the fourth light neutrino. This is realized by the so-called singular see-saw mechanism [12, 13]. The goal is achieved, by having an $m_{R}$ of rank $n_{R}-1$. However, this is not enough, we still have to fine tune $m_{R}$ to the keV-MeV range. This last unwanted problem can be circumvented by building a second stage of see-saw structure. This still fits into the scheme Eq. 1, but there are in fact three scales, not two.

Here we give a formal proof that with only two scales we can not get a fourth light neutrino. The importance of this result lies in the fact that one may be tempted to believe that the larger the mass matrix the more possibilities of choosing the masses are available, and some symmetries may help getting light sterile neutrinos. The problem is defined in the following statement:

Let $m_{R}$ be a matrix of eigenvalues real positive and greater than some $M$, and let all of the moduli of elements of $m_{D}$ be much smaller than $M$, then the spectrum of $m_{\nu}$ contains the full spectrum of $m_{R}$ with corrections of the order of $m^{2} / M \equiv \max \left(\left|\left(m_{D}\right)_{i j}\right|\right)^{2} / M$. That means that no manipulation on $m_{D}$ and/or $m_{R}$ can move a mass from the heavy $m_{R}$ matrix into the light spectrum.

The proof is a simple consequence of perturbation theory for finite matrices [14]. There, it is shown, that if we have a matrix of the form

$$
M(\beta)=M^{(0)}+\beta M^{(1)},
$$

then, every non-degenerate eigenvalue of $M^{(0)}$ gives rise to a non-degenerate eigenvalue of $M(\beta)$, being an analytic function of $\beta$ in some surrounding of 
zero. Since we are interested in the heavy spectrum, the assumption of nondegeneracy is quite general. In case of degenerate eigenvalues, we still can expand the eigenvalues in series, which however will be analytic functions with branches. We limit ourselves to the former case, but the reader should understand that the theorem holds for the general case also.

We decompose $m_{\nu}$ as

$$
\begin{aligned}
\left(\begin{array}{cc}
0 & m_{D} \\
m_{D}^{T} & m_{R}
\end{array}\right) & =\left(\begin{array}{cc}
0 & 0 \\
0 & m_{R}
\end{array}\right)+\left(\begin{array}{cc}
0 & m_{D} \\
m_{D}^{T} & 0
\end{array}\right) \\
& =M\left[\left(\begin{array}{cc}
0 & 0 \\
0 & m_{R} / M
\end{array}\right)+\beta\left(\begin{array}{cc}
0 & m_{D} / m \\
m_{D}^{T} / m & 0
\end{array}\right)\right] \\
& \equiv M\left(M^{(0)}+\beta M^{(1)}\right),
\end{aligned}
$$

where $\beta=m / M$. The first matrix has all its elements greater than one, while the second has all elements smaller than one, both are of course dimensionless. The eigenvectors of $M^{(0)}$ are of the form (we chose without loss of generality a weak base for neutrinos in which $m_{R}$ is diagonal [15])

$$
e_{i}=\left(\begin{array}{c}
0 \\
\vdots \\
1 \\
\vdots \\
0
\end{array}\right)
$$

To find the first order correction, we expand the eigenvectors as

$$
v_{i}=\sum_{j} \alpha_{j i} e_{j} .
$$

This gives us the following equation

$$
\left(M^{(0)}+\beta M^{(1)}\right) v_{i}=\lambda_{i} v_{i},
$$

which is solved into

$$
\lambda_{i}=\lambda_{i}^{(0)}+\beta \frac{1}{\alpha_{i i}} \sum_{j} \alpha_{j i} e_{i}^{T} M^{(1)} e_{j},
$$


where $\lambda^{(0)}$ are non-degenerate nonzero eigenvalues of $M^{(0)}$, which are also eigenvalues of $m_{R} / M$. Obviously, only $\alpha_{i i}$ is $\mathcal{O}(1)$, and $\alpha_{j i}$ for $j \neq i$ is $\mathcal{O}(\beta)$. The first order correction to $\lambda^{(0)}$ is therefore

$$
\lambda_{i}^{(1)}=\lambda_{i}^{(0)}+\beta e_{i}^{T} M^{(1)} e_{i} .
$$

But this vanishes due to the nondiagonal form of $M^{(1)}$. Thus the first nonvanishing correction to the large eigenvalues is of the order $M \beta^{2}=m^{2} / M$, which completes the proof. Remark, that the masses of the neutrinos are moduli of the eigenvalues of $m_{\nu}$. The corrections to the moduli are however of the same order.

Using simple arguments based on perturbation series, we have shown that a natural $m_{R}$ (no fine tunings and eigenvalues at the heavy scale) leads to three light neutrinos. Thus from the class of see-saw type models only a singular double see-saw mechanism can accommodate the LSND data and an additional fourth light neutrino state.

\section{Acknowledgments}

This work was supported by the Polish Committee for Scientific Research under Grant No. 2P03B04919 and 2P03B05418.

\section{References}

[1] T. Yanagida, Proc. of the Workshop on the Unified Theory and Baryon Number in the Universe, eds. O. Sawada and A. Sugamoto (KEK,1979), p.95; M. Gell-Mann, P. Ramond and R. Slansky, in Supergravity, ed. by P. Van Neiuwenhuizen and D. Freedman (NorthHolland,Amsterdam,1979), p.315.

[2] R. Mohapatra and G. Senjanovic, Phys. Rev. Lett., 44 (1980) 912.

[3] C. Athanassopoulos et al., Phys. Rev. Lett. 77(1996)3082; Phys. Rev. C54 (1996) 268; Phys. Rev. C58 (1998) 2489; Phys. Rev. Lett. 81 (1998) 1774; S.J. Yellin, hep-ex/9902012.

[4] T. Toshito, hep-ex/0105023. 
[5] E. Chun, A.S. Joshipura, and A.Yu. Smirnov, Phys. Lett. B 357 (1995) 608.

[6] R. Foot and R.R. Volkas, Phys. Rev. D52 (1995) 6595; Z.G. Berezhiani and R.B. Mohapatra, Phys. Rev. D 52 (1995) 6607; R.N. Mohapatra, hep-ph/9903261.

[7] E. Ma and P. Roy, Phys. Rev. D52 (1995) 4780; E. Ma, D.P. Roy and U. Sarkar, Phys. Lett. B444 (1998) 391.

[8] S.C. Gibbons, R.N. Mohapatra, S. Nandi and A. Raychaudhuri, Phys. Lett. B430 (1998) 296.

[9] U. Sarkar, Phys. Rev. D59 031301.

[10] R. N. Mohapatra, hep-ph/9910365.

[11] G.M. Fuller, J.R. Primack and Y.Z. Qian, Phys. Rev. D 52 (1995) 1288; V. Barger, T.J. Weiler, and K. Whisnant, Phys. Lett. B 427 (1998) 97; S.M. Bilenky, C. Giunti, and W. Grimus, Eur. Phys. J. C 1 (1998) 247; Phys. Rev. D 57 (1998) 1920; S.M. Bilenkii, C. Giunti, W. Grimus and T. Schwetz, Astropart. Phys. 11 (1999) 413; N. Okada and O. Yasuda, Int. J. Mod. Phys. A 12 (1997) 3669; J.M. Gelb and S.P. Rosen, Phys. Rev. D 62 (2000) 013003, V. Barger, S. Pakvasa, T.J. Weiler and K. Whisnant, Phys. Rev. D58 (1998) 093016; V. Barger, Y. Dai, K. Whisnant and B. Young, Phys. Rev. D59 (1999) 113010; R.N. Mohapatra, S. Nussinov, Phys. Rev. D60 013002; S. Mohanty, D.P. Roy, U. Sarkar, Phys. Lett. B445 (1998) 185; S. M. Bilenky, S. Pascoli and S. T. Petcov, hep-ph/0104218.

[12] E.J. Chun, C.W. Kim and U.W. Lee, Phys. Rev. D58 (1998) 093003;

[13] Y. Chikira, N. Haba and Y. Mimura, Eur. Phys. J. C 16 (2000) 701.

[14] M. Reed, B. Simon in "Methods of modern mathematical physics", Academic Press, New York, London, 1972.

[15] J. Gluza and M. Zralek, Phys. Rev. D 51 (1995) 4695. 